Paulo Frazão 1,2

Paulo Capel Narvai 1

Maria do Rosário Dias de Oliveira Latorre 1

Roberto Augusto Castellanos 1

\title{
Prevalência de oclusopatia na dentição decídua e permanente de crianças na cidade de São Paulo, Brasil, 1996
}

\author{
Malocclusion prevalence in the deciduous \\ and permanent dentition of schoolchildren \\ in the city of São Paulo, Brazil, 1996
}

1 Faculdade de Saúde Pública, Universidade de São Paulo.

Av. Dr. Arnaldo 715, São Paulo, SP 01246-904, Brasil.

2 Faculdade de Odontologia, Universidade Metodista de São Paulo.

Rua do Sacramento 230, São Bernardo do Campo, SP 09735-460, Brasil.

\begin{abstract}
The purpose of this study was to estimate the prevalence of malocclusion in deciduous and permanent dentition of schoolchildren enrolled in public and private schools in the city of Sao Paulo, Brazil, in 1996. Occlusal conditions were classified as normal, mild, and moderate/severe according to World Health Organization criteria. The results refer to 985 schoolchildren ages five and 12 years. Prevalence of malocclusion was high, increasing from $48.97 \pm 4.53 \%$ in the deciduous dentition to $71.31 \pm 3.95 \%$ in the permanent dentition. The proportion of moderate/severe malocclusion was nearly twice as great in the second dentition $(O R=1.87 ; 95 \% C I=1.43$ 2.45; $p<0.001)$. No significant difference was observed according to gender or public vs. private school. Statistically significant differences were associated with ethnicity, indicating the complexity and diversity of occlusion in the population and suggesting the need for longitudinal studies.
\end{abstract}

Key words Public Health Dentistry; Malocclusion; Dentition; Child Health; Ethnic Groups

Resumo O objetivo deste estudo foi estimar a prevalência de oclusopatia na dentição decídua e permanente de crianças de escolas públicas e privadas do Município de São Paulo, SP, Brasil, em 1996. A condição oclusal foi classificada em três categorias: normal, leve e moderada/severa conforme os critérios da Organização Mundial da Saúde. Os resultados referem-se a 985 exames em crianças de 5 e 12 anos de idade. A prevalência das oclusopatias foi alta, aumentando de 48,97 \pm 4,53\% na dentição decídua a 71,31 \pm 3 ,95\% na dentição permanente, sendo que a proporção de oclusopatia moderada/severa foi quase duas vezes maior na dentição permanente (OR = 1,87; IC95\% = 1,43-2,45; $p<0,001$ ). Quanto ao sexo e ao tipo de estabelecimento de ensino, não foram observadas diferenças estatisticamente significativas nas idades estudadas. Diferenças estatísticas associadas aos grupos étnicos denotam a complexidade e diversidade da oclusão na população e sugerem que estudos longitudinais devem ser realizados.

Palavras-chave Odontologia em Saúde Pública; Maloclusão; Dentição; Saúde Infantil; Grupos Étnicos 


\section{Introdução}

Os problemas de oclusão dentária denominados de oclusopatias, conforme nomenclatura empregada por Simões (1978), consistem de anomalias do crescimento e desenvolvimento, afetando, principalmente, os músculos e os ossos maxilares no período da infância e da adolescência, que podem produzir alterações tanto do ponto de vista estético nos dentes e/ou face, quanto do ponto de vista funcional na oclusão, mastigação e fonação. Esses problemas são resultantes da interação de variáveis relacionadas à hereditariedade e ao meio ambiente, incluindo os estímulos positivos e nocivos presentes principalmente durante a formação e desenvolvimento do complexo orofacial na infância e adolescência (Planas, 1987; Van der Linden, 1966). A disposição dos dentes nos arcos dentários, a forma e o volume dos ossos maxilares, a maneira pela qual se articulam os músculos e as articulações envolvidas não permanecem estáticas durante toda a vida, mudando continuamente em resposta aos processos de crescimento, influências do meio ambiente, tratamentos dentários, desgastes, processos patológicos e envelhecimento.

Estudos antropológicos mostraram que pesadas solicitações no sistema mastigatório não são características dos habitantes das cidades modernas. Não surpreende, portanto, que as dentições de muitas populações hodiernas, comparadas àquelas de seus ancestrais, que eram caçadores e juntavam alimentos de forma nômade, denotem evidência de reduzida função e eficiência mastigatória, juntamente com uma crescente prevalência de oclusopatias (Brown, 1985; Varrela, 1990; Villavicencio et al., 1996).

Embora muito das informações epidemiológicas seja resultado da análise de estudos transversais, incluindo os possíveis vieses inerentes a essas pesquisas, reconhece-se que a prevalência e a severidade das oclusopatias têm aumentado nos últimos 200 anos, especialmente o apinhamento dentário (Brin et al., 1998; Corruccini, 1984; De Muelenaere et al., 1992; Weiland et al., 1997).

A Organização Mundial da Saúde (OMS) recomenda às autoridades sanitárias a realização de levantamentos epidemiológicos das principais doenças bucais nas idades de 5, 12 e 15 anos e nas faixas etárias de 35-44 e 65-74 anos, numa periodicidade entre cinco e dez anos. $\mathrm{O}$ conhecimento da situação de saúde bucal de diferentes grupos populacionais, por meio de levantamentos epidemiológicos, é fundamental para o desenvolvimento de propostas de ações adequadas às suas necessidades e riscos, bem como para a possibilidade de comparações que permitam avaliar o impacto dessas ações.

O objetivo deste estudo foi estimar a prevalência das oclusopatias na dentição decídua e permanente de crianças em escolas públicas e privadas do Município de São Paulo, São Paulo, em 1996, e investigar possíveis associações com sexo, tipo de escola e grupo étnico.

\section{Material e método}

O material analisado foi uma base de dados secundários proveniente de uma amostra probabilística por conglomerados obtida no Levantamento Epidemiológico da Cárie Dentária, Oclusopatias e Fluorose Dentária, em Crianças de 5 a 12 Anos de Idade, em Escolas Públicas e Privadas do Município de São Paulo. Esta pesquisa foi um estudo transversal mais abrangente realizado pela Faculdade de Saúde Pública (FSP) da Universidade de São Paulo (USP) em parceria com a Secretaria de Estado da Saúde de São Paulo (FSP-USP, 1997) no qual as unidades amostrais foram escolas e pré-escolas públicas e particulares distribuídas por todas as regiões da cidade, totalizando 103 unidades amostrais, de onde foram obtidos 2.491 elementos amostrais nas idades de 5 a 12 anos em ambos os sexos.

Para este estudo, foram consideradas apenas crianças de 5 e de 12 anos de idade, a fim de permitir a comparação das condições oclusais nas dentições decídua e permanente correspondentes, respectivamente, a 5 e 12 anos. Admitindo-se uma proporção de problemas oclusais igual a $50 \%$ na população dessas idades, um nível de confiança igual a 95\% e uma precisão de $\pm 5 \%$, considerou-se que o tamanho da amostra é adequado para produzir inferências para a população dessas idades do município como um todo (Lwanga \& Lemeshow, 1991).

A variável grupo étnico, determinada com base em traços físicos, também foi incluída na análise, sendo adotadas as categorias pertinentes à população paulistana preconizadas pela Fundação Instituto Brasileiro de Geografia e Estatística (IBGE), utilizadas no Censo Populacional de 1991: amarela, branca, negra e parda. Para a classificação dos escolares pelos examinadores foram utilizados os seguintes critérios: a) Amarela: pele branco-amarela; olhos oblíquos, repuxados;

b) Branca: pele branca; cabelo liso ou ondulado fino (de louro a negro); nariz estreito e proeminente; lábios finos (ou de espessura mediana); gengiva cor rósea (com suas variações nor- 
mais em virtude da queratinização e vascularização);

c) Negra: pele castanho-escura ou negra; cabelo ondulado, encarapinhado ou em anel, geralmente escuro; nariz largo ou achatado; gengiva pigmentada pelo acúmulo de melanina; d) Parda: pele de coloração entre branca e negra ("mulato", "moreno"); traços evidenciando miscigenação; impossibilidade de incluir o indivíduo nas categorias "branco", "negro" ou "amarelo".

A condição oclusal foi avaliada em três categorias, conforme critérios preconizados pela OMS em 1987 (OMS, 1991): normal, leve - quando há um ou mais dentes com giroversão ou ligeiro apinhamento ou espaçamento prejudicando o alinhamento regular - e moderada/severa - quando há um efeito inaceitável sobre a aparência facial, ou uma significativa redução da função mastigatória, ou problemas fonéticos observados pela presença de uma ou mais das seguintes condições nos quatro incisivos anteriores:

a) Transpasse horizontal maxilar estimado em 9mm ou mais;

b) Transpasse horizontal mandibular, mordida cruzada anterior igual ou maior que o tamanho de um dente;

c) Mordida aberta;

d) Desvio de linha média estimado em $4 \mathrm{~mm}$ ou mais;

e) Apinhamento ou espaçamento estimado em $4 \mathrm{~mm}$ ou mais.

Cabe ressaltar que as alterações oclusais não explicitadas nos critérios mencionados, como, por exemplo, mordida cruzada posterior (uni ou bilateral), sobremordida ou transpasse vertical acima de $2 \mathrm{~mm}$, foram incluídas na categoria leve.

O erro interexaminadores aferido durante a calibração foi de $26,7 \%$, e a discordância intra- examinadores, calculada a partir de 163 reexames $(6,5 \%$ do total), foi de $4,9 \%$. Embora na estatística de proporções o erro interexaminadores tenha alcançado uma cifra um pouco acima da recomendada, considerou-se que, à semelhança de outros instrumentos epidemiológicos, grande parte desse erro ocorreu em função da dificuldade de distinção entre as categorias normal e leve. Como o núcleo central da análise refere-se à presença ou não de oclusopatia moderada/severa e como resultados acidentais dificilmente ocorrem diante de um grande número de observações, considerou-se que essa limitação do instrumento de medida não prejudicou a confiabilidade dos resultados obtidos, tendo em vista o tamanho da amostra $(\mathrm{n}=985)$.

As prevalências estimadas foram representadas como $\hat{p} \pm 1,96$. EP $\hat{p}$, onde EP $\hat{p}$ é o erro padrão da proporção estimada. A análise estatística univariada foi efetuada com auxílio da versão 6.02 do programa Epi Info (CDC/WHO, 1990), na qual se verificou a associação entre as variáveis pelo cálculo do qui-quadrado com correção de Yates e da odds ratio, sendo adotado o nível de significância de $5 \%$.

\section{Resultados}

Os resultados referem-se a 985 exames. Na Tabela 1 é apresentada a distribuição da amostra segundo o tipo de dentição, o sexo e o tipo de escola, observando-se homogeneidade quanto à composição dos elementos segundo essas variáveis.

Em relação ao tipo de dentição, observouse uma freqüência maior de problemas oclusais na dentição permanente $(71,31 \pm 3,95 \%)$ escolares de 12 anos de idade - do que na dentição decídua $(48,97 \pm 4,53 \%)$ - crianças de 5

Tabela 1

Distribuição da amostra segundo a dentição e o sexo por tipo de estabelecimento de ensino. São Paulo, 1996.

\begin{tabular}{|c|c|c|c|c|c|}
\hline \multirow[t]{3}{*}{ Estratos } & \multicolumn{4}{|c|}{ Dentição* } & \multirow[t]{3}{*}{ Total } \\
\hline & \multicolumn{2}{|c|}{ Decídua } & \multicolumn{2}{|c|}{ Permanente } & \\
\hline & Masculino & Feminino & Masculino & Feminino & \\
\hline Pré-escola pública & 119 & 126 & - & - & 245 \\
\hline Pré-escola privada & 122 & 123 & - & - & 245 \\
\hline Escola pública de ensino fundamental & - & - & 121 & 136 & 257 \\
\hline Escola privada de ensino fundamental & - & - & 121 & 117 & 238 \\
\hline Total & 241 & 249 & 242 & 253 & 985 \\
\hline
\end{tabular}

* Dentição decídua observada aos 5 anos e permanente aos 12 anos de idade. 
anos. A proporção da população com oclusão normal sofreu queda acentuada com a idade no período de transição da dentição $(\mathrm{p}<0,001)$. Enquanto na dentição decídua a proporção da população com problemas oclusais é semelhante à proporção com oclusão normal, na dentição permanente esta relação é 2,5 vezes maior. O crescimento da proporção de condição oclusal leve é semelhante ao aumento da proporção de condição moderada/severa, de 22,96 \pm $3,65 \%$ para $31,52 \pm 4,08 \%$ e de $26,12 \pm 3,84 \%$ para $39,79 \pm 4,34 \%$, respectivamente, nos dois tipos de dentição (Figura 1). A proporção de oclusopatia moderada/severa foi quase duas vezes maior na dentição permanente $(\mathrm{OR}=1,87$; IC95\% = 1,43-2,45; $\mathrm{p}<0,001)$.

Quanto ao sexo e ao tipo de escola, não foram observadas diferenças estatisticamente significativas nas idades estudadas. Mais da metade das crianças examinadas foram incluídas no grupo étnico branco. A Tabela 2 mostra a distribuição da amostra segundo a etnia.

A condição oclusal nas dentições decídua e permanente segundo o grupo étnico é apresentada nas Figuras 2 e 3, respectivamente. Pode-se observar que essa variável provocou diferenças na proporção das categorias relacionadas à condição oclusal tanto na dentição decídua quanto na permanente. No entanto, essas diferenças não foram consistentes para produzir medidas estatisticamente significativas,

\section{Figura 1}

Distribuição percentual dos escolares segundo a condição oclusal e o tipo de dentição. São Paulo, 1996.

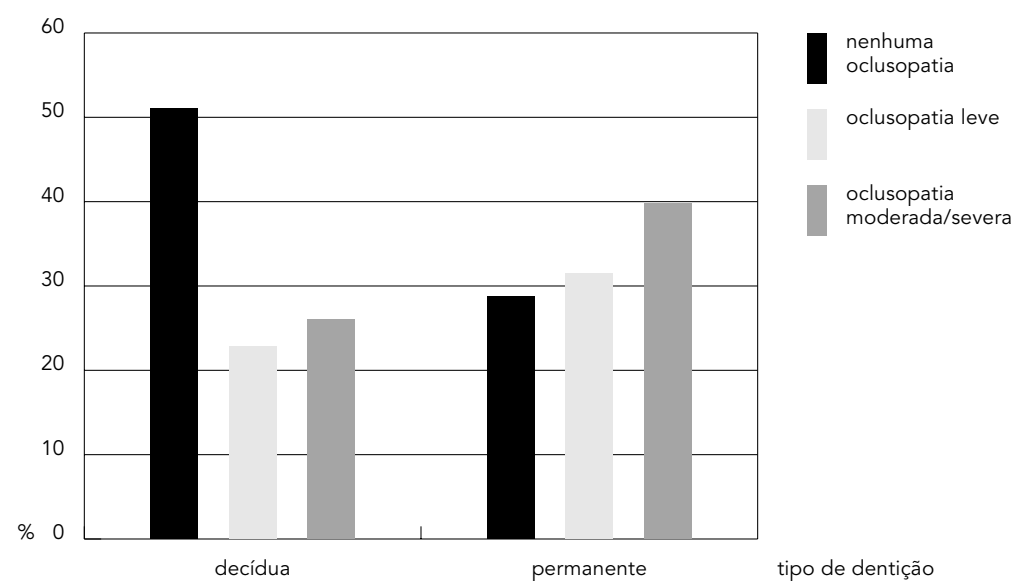

$\chi^{2}=51,6 ; p<0,001$ $\mathrm{p}=0,07$ e $\mathrm{p}=0,11$, respectivamente, aos 5 anos e 12 anos de idade.

Na Tabela 3 é mostrada a análise univariada, desagregando os dados por tipo de dentição e combinando as categorias oclusão normal e oclusopatia leve. Verificou-se associação com grupo étnico tanto na dentição decídua $(\mathrm{p}=0,05)$ quanto na permanente $(\mathrm{p}<0,05)$, no entanto em sentidos completamente antagônicos. Enquanto na primeira dentição notou-se uma associação positiva com oclusopatia moderada/severa $(O R=1,81 ; p<0,05)$, na segunda encontrou-se uma associação negativa $(\mathrm{OR}=$ $0,62 ; \mathrm{p}<0,05)$.

\section{Discussão}

\section{Prevalência e severidade}

$\mathrm{Na}$ análise da ocorrência das oclusopatias deve-se considerar que o Município de São Paulo, com uma população de cerca de 10 milhões de habitantes em 1995 (densidade demográfica = $6.624,7 \mathrm{hab} / \mathrm{km}^{2}$ ), apresenta características gerais de morbi-mortalidade que o situam em um perfil de "transição epidemiológica", em que coexistem elevados coeficientes de mortalidade por doenças típicas tanto dos países considerados subdesenvolvidos quanto dos países desenvolvidos. O coeficiente de mortalidade infantil, por exemplo, variou, em 1999, de 5,04 por mil nascidos vivos (distrito da Lapa) até 28,57 por mil (distrito de Marsilac).

Do ponto de vista da saúde bucal, existe na cidade um sistema de prevenção (Frazão \& Narvai, 1996), com participação tanto de instituições públicas quanto de empresas privadas, cujo eixo central é a fluoretação das águas de abastecimento público, iniciada em outubro de 1985. O Sistema de Vigilância Sanitária dos Teores de Flúor nas Águas de Abastecimento Público, em operação desde 1990, permite estabelecer um quadro consistente e confiável, tanto quanto à abrangência da medida quanto em relação aos teores efetivamente oferecidos ao consumo da população. Assim, é possível afirmar que a queda dos índices de cárie dentária na população infantil identificada em 1996 (índice CPOD aos 12 anos = 2,06 \pm 0,21), indicando uma situação de baixa prevalência e atingindo a meta da OMS para o ano 2000 - índice CPOD aos 12 anos $\leq 3,0$ é devida, em grande parte, a essa medida de prevenção (Narvai et al., 2000). Este aspecto (baixa prevalência de cárie) provavelmente influenciou os resultados obtidos, tendo em vista que a perda precoce de dentes decíduos e permanentes constitui cau- 
sa importante de problemas oclusais. Como tais perdas foram praticamente nulas em 1996, é lícito admitir o impacto positivo que esses baixos níveis de cárie dentária exerceram na prevalência de oclusão normal.

A prevalência das oclusopatias na população infantil foi alta, crescendo de 49,0 $\pm 4,53 \%$ na dentição decídua ( 5 anos de idade) para $71,3 \pm 3,95 \%$ na dentição permanente (12 anos). Apesar das diferenças metodológicas, resultados semelhantes foram encontrados no Brasil, para a idade de 12 anos, por Bello (1987, apud Galvão et al., 1994) em Porto Alegre, Galvão \& Silva (1990) em Araçatuba e Martildes et al. (1992) em São José dos Campos; na América Latina, por Saturno (1980) em Caracas, Venezuela, Ibarra et al. (1985) em Havana e Torres \& Corrales (1985) em Camaguey, cidades de Cuba; na Europa, por Trombelli et al. (1991) em Argenta, Itália. Em relação à proporção de oclusopatia moderada/severa, resultados semelhantes foram observados por Masztalerz (1989), Burden \& Holmes (1994) e O’Dowling \& O'Mullane (1995). Suszczewicz (1994) apresentou resultados inferiores. Cabe destacar ainda, que a baixa proporção de condição oclusal normal $(28,7$ $\pm 3,91 \%$ ) na dentição permanente verificada neste estudo é semelhante à encontrada por Silva Filho et al. (1990), muito embora este autor tenha pesquisado a dentição mista de 2.416 crianças de escolas públicas e privadas da ci- dade de Bauru, São Paulo. Na idade de 5 anos, os resultados são iguais aos observados por Viskovic et al. (1990), Kabue et al. (1995), Tomita (1996) e Legovic et al. (1998).

O tipo de escola (pública ou privada) e o sexo não influenciaram a freqüência das oclusopatias, corroborando estudo de Tomita (1996). No entanto, o tipo de dentição, relacionandose a idade de 5 anos com dentição decídua e a idade de 12 anos com a permanente, mostrou forte associação ( $\mathrm{p}<0,001)$, sendo que a proporção de problemas oclusais foi cerca de 1,5 vez maior na dentição permanente do que na

Tabela 2

Número e percentual da amostra segundo o grupo étnico por tipo de dentição. São Paulo, 1996.

\begin{tabular}{|c|c|c|c|c|c|c|}
\hline \multirow[t]{3}{*}{ Grupo étnico } & \multicolumn{4}{|c|}{ Dentição* } & \multicolumn{2}{|c|}{ Total } \\
\hline & \multicolumn{2}{|c|}{ Decídua } & \multicolumn{2}{|c|}{ Permanente } & \multirow[b]{2}{*}{$n$} & \multirow[b]{2}{*}{$\%$} \\
\hline & $n$ & $\%$ & $n$ & $\%$ & & \\
\hline Amarelo & 10 & 2,0 & 16 & 3,2 & 26 & 2,6 \\
\hline Branco & 307 & 62,7 & 258 & 52,1 & 565 & 57,4 \\
\hline Negro & 32 & 6,5 & 29 & 5,9 & 61 & 6,2 \\
\hline Pardo & 141 & 28,8 & 192 & 38,8 & 333 & 33,8 \\
\hline Total & 490 & 100,0 & 495 & 100,0 & 985 & 100,0 \\
\hline
\end{tabular}

* Dentição decídua observada aos 5 anos e permanente aos 12 anos de idade.

Figura 2

Distribuição percentual dos escolares de 5 anos de idade segundo a condição oclusal

e o grupo étnico. São Paulo, 1996.

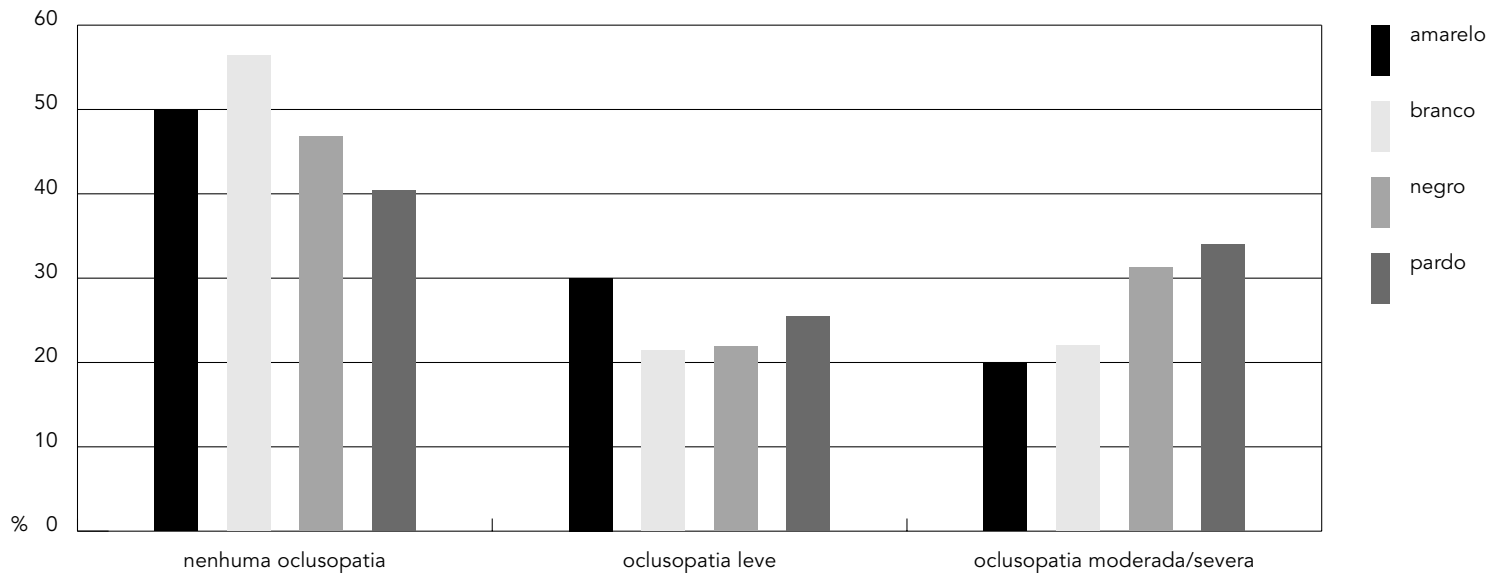

$\chi^{2}=11,55 p=0,07$ 
Figura 3

Distribuição percentual dos escolares de 12 anos de idade segundo a condição oclusal

e o grupo étnico. São Paulo, 1996.

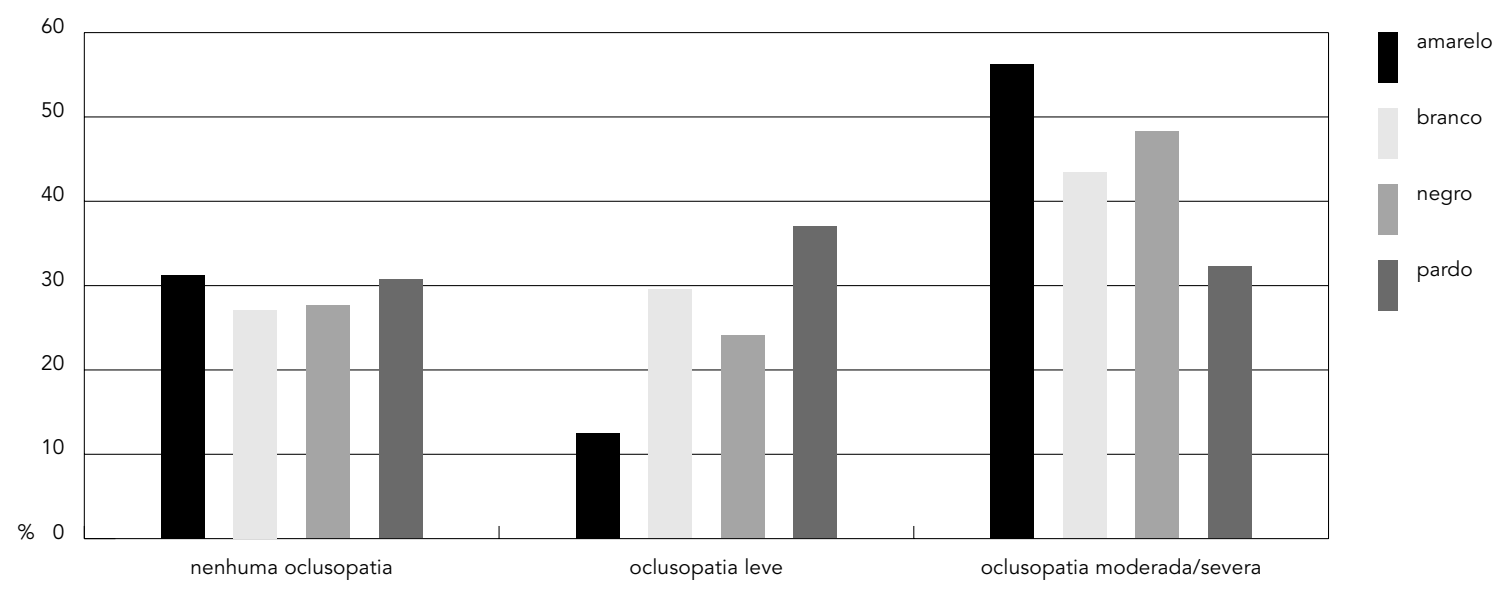

$\chi^{2}=10,22 ; p=0,11$

Tabela 3

Oclusão normal e oclusopatia leve versus oclusopatia moderada/severa.

Análise univariada segundo o tipo de dentição e o grupo étnico.

\begin{tabular}{|c|c|c|c|c|c|c|}
\hline \multirow[t]{2}{*}{$\begin{array}{l}\text { Dentição/ } \\
\text { Grupo étnico }\end{array}$} & \multicolumn{3}{|c|}{$\begin{array}{c}\text { Oclusopatia } \\
\text { moderada/severa }\end{array}$} & \multirow[t]{2}{*}{ Odds ratio } & \multirow[t]{2}{*}{$p\left(\chi^{2}\right)$} & \multirow[t]{2}{*}{$\chi^{2}$} \\
\hline & + & - & Total & & & \\
\hline \multicolumn{7}{|l|}{ Decídua } \\
\hline negro & 10 & 22 & 32 & 1,60 & 0,05208 & 7,72 \\
\hline pardo & 48 & 93 & 141 & $1,81^{*}$ & & \\
\hline amarelo & 2 & 8 & 10 & 0,88 & & \\
\hline branco & 68 & 239 & 307 & 1,00 & & \\
\hline \multicolumn{7}{|l|}{ Permanente } \\
\hline negro & 14 & 15 & 29 & 1,22 & 0,03514 & 8,6 \\
\hline pardo & 62 & 130 & 192 & $0,62^{*}$ & & \\
\hline amarelo & 9 & 7 & 16 & 1,68 & & \\
\hline branco & 112 & 146 & 258 & 1,00 & & \\
\hline
\end{tabular}

${ }^{*} p<0,05$. porção da população com oclusão normal e, de outro, reduzir o percentual de oclusão moderada/severa para níveis mais suportáveis economicamente e aceitáveis socialmente.

\section{Etnia}

Nesta pesquisa verifica-se que, de modo geral, a distribuição proporcional das categorias da variável etnia é semelhante ao observado no Censo Populacional de 1991 e na Pesquisa Nacional por Amostra de Domicílios de 1993 (IBGE, 1996), com uma ligeira diferença relacionada à menor proporção de brancos e maior freqüência relativa de pardos. Na idade de 5 anos, as estimativas $(62,7 \%$ de brancos e $28,8 \%$ de pardos) são mais próximas às do censo e da Pesquisa Nacional por Amostra de Domicílio do que no estrato correspondente à segunda dentição (52,1\% de brancos e 38,8\% de pardos). A comparação apresentada na Tabela 4 permite apreciar a qualidade das estimativas produzidas e atestar sua validade.

Os dados obtidos na presente investigação mostraram resultados que se caracterizaram pela ambigüidade. Enquanto na análise univariada de ambas as dentições, a etnia não apresentou associação consistente com oclusopatia moderada/severa $(\mathrm{p}>0,05)$, na análise por tipo de dentição, observou-se em relação à categoria pardo tanto associação positiva $(\mathrm{OR}=$ 
$1,81 ; \mathrm{p}<0,05)$ com problemas oclusais severos (dentição decídua), quanto associação negativa notada aos 12 anos de idade $(\mathrm{OR}=0,62 ; \mathrm{p}<$ $0,05)$. Essa ambigüidade verificada na comparação das categorias branco e pardo pode estar relacionada, entre outros aspectos, à atribuição das categorias da variável a cada criança examinada e ao caráter de indeterminação que essa variável parece assumir numa população com traços étnicos tão diversificados como a da cidade de São Paulo.

Os resultados mostraram que a probabilidade de oclusopatia moderada/severa não foi diferente entre os grupos étnicos branco e pardo para o conjunto da amostra. Tomando por base os intervalos de confiança das proporções em cada categoria, Silva \& Araújo (1983), estudando escolares de 5 a 7 anos no Rio de Janeiro, e Tomita (1996), pesquisando a oclusão de crianças de 3 a 5 anos na cidade de Bauru, São Paulo, também não encontraram diferenças estatisticamente significativas entre essas categorias étnicas e a presença de má-oclusão.

Embora a relação entre certos traços físicos e o padrão facial, muito notavelmente àqueles tipos associados à relação anterior-posterior dos maxilares, seja largamente reconhecida na literatura (Baume, 1974; Brunelle et al., 1996; El-Mangoury \& Mostafa, 1990; Emrich et al., 1965; Grecco, 1966; Kelly et al., 1973; Kelly \& Harvey, 1977; Lew et al., 1993; Proffit et al., 1998; Takahashi, 1975; Trottman \& Elsbach, 1996; Wheeler et al., 1994), não existe conhecimento suficiente para sustentar a noção apresentada por Van der Linden (1966), de que o aumento da prevalência das oclusopatias seja atribuível à miscigenação racial/étnica, sendo esta responsável pela elevação considerável da variabilidade genética com a sucessão das gerações. As contribuições científicas contemporâneas, oriundas principalmente da genética e da antropologia, apontam para direção oposta.

Os estudos de evolução humana vêm rejeitando a suposição de que algumas características externas visíveis (por exemplo, a cor da pele) expressariam uma medida consistente representativa de outras características de um indivíduo ou população. Embora essa suposição persista, estima-se que essas características representam somente $0,01 \%$ dos cerca de $100 \mathrm{mil}$ genes de que cada pessoa é dotada. Conforme a moderna teoria genética relativa às características poligênicas, as diferenças de freqüência de genes entre grupos das mais distintas etnias não são significativas. Estudos objetivando compreender as fontes da variação humana revelaram que aproximadamente $85 \%$ de toda a variação na freqüência genética ocorrem

\begin{tabular}{|c|c|c|c|c|c|c|}
\hline \multicolumn{7}{|c|}{$\begin{array}{l}\text { Número e percentual das estimativas segundo o grupo étnico, } \\
\text { a pesquisa e o ano de realização. }\end{array}$} \\
\hline \multirow[t]{3}{*}{ Grupo étnico } & \multicolumn{6}{|c|}{ Pesquisa e ano de realização } \\
\hline & \multicolumn{2}{|c|}{ Amostraa (1996) } & \multicolumn{2}{|c|}{ Censob (1991) } & \multicolumn{2}{|c|}{ PNADc (1993) } \\
\hline & $\mathrm{n}$ & $\%$ & $\mathrm{n}$ & $\%$ & $\mathrm{n}$ & $\%$ \\
\hline Amarelo & 26 & 2,6 & 206.702 & 2,1 & 334.643 & 2,1 \\
\hline Branco & 565 & 57,4 & 6.665 .960 & 69,1 & 11.168 .188 & 69,9 \\
\hline Negro & 61 & 6,2 & 371.042 & 3,8 & 623.785 & 3,9 \\
\hline Pardo & 333 & 33,8 & 2.281 .067 & 23,6 & 3.838 .541 & 24,0 \\
\hline Indígena & - & - & 4.690 & 0,2 & 8.743 & 0,1 \\
\hline Ignorado & - & - & 116.724 & 1,2 & - & - \\
\hline Total & 985 & 100,0 & 9.646 .185 & 100,0 & 15.973 .900 & 100,0 \\
\hline
\end{tabular}

a Pesquisa da Faculdade de Saúde Pública, Universidade de São Paulo/ Secretaria Estadual de Saúde de São Paulo: cidade de São Paulo.

b Censo populacional: cidade de São Paulo.

c Pesquisa nacional por amostragem de domicílios: região metropolitana de São Paulo.

dentro dos mesmos grupos populacionais, e somente $15 \%$ são observados entre as populações com traços físicos reconhecidamente diferentes. Assim, do ponto de vista da genética, considerar os traços físicos mais marcantes como um conceito biológico que traduz populações geneticamente homogêneas é insustentável e não encerra mais legitimidade no campo das ciências biológicas (Freeman, 1998; Pena et al., 2000; Templeton, 1998).

Neste sentido, admite-se que a maior parte das variações oclusais seja resultado da interação de fatores ambientais e genéticos em cada grupo populacional do que entre os diferentes grupos populacionais.

Finalmente, os resultados observados na presente investigação revelaram a necessidade de estudos longitudinais e a importância cada vez maior da antropologia e da genética nas investigações dos fenômenos de crescimento e desenvolvimento cranio-maxilo-facial, principalmente quando se focalizam populações da América Latina, cuja origem e formação são das mais complexas e diversificadas do planeta, predominando em determinadas regiões, conforme Galvão et al. (1994), ora miscigenação tri-híbrida ora bi-híbrida. A população da cidade de São Paulo expressa, em suas características de oclusão, essa complexidade e diversidade poliétnica. 


\section{Agradecimentos}

Os autores agradecem a todos que, direta e indiretamente, contribuíram para a realização da pesquisa que deu origem ao banco de dados, tanto no âmbito da Secretaria de Estado da Saúde de São Paulo como no da Faculdade de Saúde Pública da Universidade de São Paulo, bem como aos estabelecimentos de ensino públicos e privados. A Professora M. R. D. O. Latorre agradece ao Conselho Nacional de Desenvolvimento Científico e Tecnológico a bolsa de pesquisador (processo no 300318/97-9).

\section{Referências}

BAUME, L. J., 1974. Uniform methods for the epidemiologic assessment of malocclusion. Results obtained with the World Health Organization standard methods (1962 and 1971) in South Pacific Populations. American Journal of Orthodontics, 66:251-272.

BROWN, T., 1985. Desenvolvimento e função oclusal nos aborígenes australianos. In: Ortopedia Funcional dos Maxilares Vista Através da Reabilitação Neuro-Oclusal (W. A. Simões, org.), pp. 1-67, São Paulo: Editora Santos.

BRIN, I.; ZWILLING-SELLAM, O.; HARARI, D.; KOYOUMDJISKY-KAYE, E. \& BEN-BASSAT, Y., 1998. Does a secular trend exist in the distribution of occlusal patterns? Angle Orthodontist, 68:81-84.

BRUNELLE, J. A.; BHAT, M. \& LIPTON, J. A., 1996. Prevalence and distribution of selected occlusal characteristics in the US population, 1988-1991. Journal of Dental Research, 75:706-713.

BURDEN, D. J. \& HOLMES, A., 1994. The need for orthodontic treatment in the child population of the United Kingdom. European Journal Orthodontics, 16:395-399.

CDC (Centers for Disease Control and Prevention)/ WHO (World Health Organization), 1990. Epi Info. Epidemiologia em Microcomputadores: Um Sistema de Processamento de Texto, Banco de Dados e Estatísticas. Atlanta: CDC/WHO.

CORRUCCINI, R. S., 1984. An epidemiologic transition in dental occlusion in world populations. American Journal of Orthodontics, 86:419-426.

DE MUELENAERE, J. J. G. G.; WILTSHIRE, W. A. \& VILJOEN, W. P., 1992. The occlusal status of an urban and a rural Venda group. Journal of the Dental Association of South African, 47:517-520.

EL-MANGOURY, N. H. \& MOSTAFA, Y. A., 1990. Epidemiologic panorama of dental occlusion. Angle Orthodontics, 60:207-213.

EMRICH, R. E.; BRODIE, A. G. \& BLAYNEY, J. R., 1965. Prevalence of Class I, Class II, and Class III malocclusions (Angle) in an urban population: An epidemiological study. Journal of Dental Research, 44:947-953.
FRAZÃO, P. \& NARVAI, P. C., 1996. Promoção da saúde bucal em escolas. In: Disciplina de Odontologia Preventiva e Saúde Pública: Manual do Aluno (Departamento de Prática de Saúde Pública, Faculdade de Saúde Pública - FSP -, Universidade de São Paulo - USP -, org.), pp. 1-28, São Paulo: FSP, USP.

FREEMAN, H. P., 1998. The meaning of race in science: Considerations for cancer research. Cancer, 82:219-225.

FSP-USP (Faculdade de Saúde Pública - Universidade de São Paulo), 1997. Levantamento Epidemiológico da Cárie Dentária, Oclusopatias e Fluorose Dentária, em Crianças de 5 a 12 Anos de Idade, em Escolas Públicas e Privadas do Município de São Paulo, em 1996. São Paulo: FSP-USP/Secretaria Estadual de Saúde de São Paulo.

GALVÃO, C. A. A. N.; PEREIRA, C. B. \& BELLO, D. R. M., 1994. Prevalência de maloclusões na América Latina e considerações antropológicas. Ortodontia, 27:52-59.

GALVÃO, C. A. A. N. \& SILVA, W. B., 1990. Prevalência de maloclusões: Estudo em escolares da cidade de Araçatuba. Ortodont Paranaen, 11:79-83.

GRECCO, A. J. T., 1966. Contribuição para o Estudo da Prevalência de Anormalidades de Oclusão em Escolares do Município de Araraquara. Amarelos Nascidos no Japão, Amarelos Nascidos no Brasil (Nisseis) e Brancos Nascidos no Brasil: Suas Relações com a Higiene Oral e as Gengivites. Dissertação de Mestrado, Araraquara: Faculdade de Odontologia de Araraquara, Universidade Estadual Paulista.

IBARRA, B. R.; BETANCOUR, E. E.; PANEQUE, A. R.; FERNANDES, S. P. \& RAMOS, F. J. A., 1985. Prevalencia de las alteraciones dentofaciales en adolescentes de la provincia ciudad de La Habana. Revista Cubana de Estomatología, 22:207-211.

IBGE (Fundação Instituto Brasileiro de Geografia e Estatística), 1996. Pesquisa Nacional por Amostra de Domicílios - 1993. Região Metropolitana de São Paulo. Rio de Janeiro: IBGE.

KABUE, M. M.; MORACHA, J. K. \& NG'ANG'A, P. M., 
1995. Malocclusion in children aged 3-6 years in Nairobi, Kenya. East African Medicine Journal, 72:210-212.

KELLY, J. E. \& HARVEY, C. R., 1977. An Assessment of the Oclusion of the Teeth of Youths 12-17 Years. US Public Heath Service, Series 11, n. 162. Washington, DC: National Center for Health Statistics.

KELLY, J. E.; SANCHEZ, M. \& van KIRK, L. E., 1973. An Assessment of the Oclusion of the Teeth of Children. US Public Heath Service, Series 11, n. 130. Washington, DC: National Center for Health Statistics.

LEGOVIC, M.; MADY, L.; FERRERI, S.; BREKALO, I.; ZUPAN, M.; MADY, B. \&VANCURA, I., 1998. Malocclusioni in dentizione decidua. Mondo Ortodontico, 23:31-36.

LEW, K. K.; FOONG, W. C. \& LOH, E., 1993. Malocclusion prevalence in an ethnic Chinese population. Australian Dental Journal, 38:442-449.

LWANGA, S. K. \& LEMESHOW, S., 1991. Sample Size Determination in Health Studies: A Practical Manual. Geneva: World Health Organization.

MARTILDES, M. L. R.; CASTELLANOS, R. A. \& ROBLES, L. P., 1992. Prevalência de mal-oclusão em escolares de 12 anos de idade de São José dos Campos. Revista Brasileira de Saúde do Escolar, 2:112-116.

MASZTALERZ, A., 1989. Frequency of indications to orthodontic treatment. Czasopismo Stomatologiczne, 42:347-349.

NARVAI, P. C.; CASTELLANOS, R. A. \& FRAZÃO, P., 2000. Prevalência de cárie em dentes permanentes de escolares do município de São Paulo, SP, 1970-1996. Revista de Saúde Pública, 34:196-200.

O'DOWLING, I. B. \& O'MULLANE, D. F., 1995. Orthodontic treatment need in the Southern Health Board. Journal of the Ireland Dental Association, 41:38-41.

OMS (Organização Mundial da Saúde), 1991. Levantamento Epidemiológico Básico de Saúde Bucal: Manual de Instruções. 3a Ed. São Paulo: Editora Santos.

PENA, S. D. J.; CARVALHO-SILVA, D. R.; ALVES-SILVA, J.; PRADO, V. F. \& SANTOS, F. R., 2000. Retrato molecular do Brasil. Ciência Hoje, 27:16-25.

PLANAS, P., 1987. Rehabilitación Neuro-Oclusal (RNO). Barcelona: Salvat.

PROFFIT, W. R.; FIELDS, H. W. \& MORAY, L. J., 1998. Prevalence of malocclusion and orthodontic treatment need in the United States: Estimates from the NHANES III survey. International Journal of Adult Orthodontics and Orthognatic Surgery, 13:97-106.

SATURNO, L. D., 1980. Características de la oclusión de 3.630 escolares del area metropolitana de Caracas. Acta Odontológica Venezolana, 18:237-261.

SILVA, C. H. T. \& ARAÚJO, T. M., 1983. Prevalência de más oclusões em escolares na Ilha do Governador, Rio de Janeiro. Parte 1: Classes I, II e III e mordida cruzada. Ortodontia, 16:10-16.

SILVA FILHO, O. G.; FREITAS, S. F. T. \& CAVASSAN, A. O., 1990. Prevalência de oclusão normal e mal-oclusão em escolares da cidade de Bauru (São Paulo). Parte II: Influência da estratificação sócio-econômica. Revista de Odontologia da Universidade de São Paulo, 4:189-196.
SIMÕES, W. A., 1978. Prevenção das oclusopatias. Ortodontia, 11:117-125.

SUSZCZEWICZ, A., 1994. Stomatologic care requirement for 12-year old children in Poland. Annales Academiae Medicae Stetinensis, 40:117-131.

TAKAHASHI, T., 1975. Prevalência de Oclusão Normal e Maloclusão em Mestiços (Xantodermas Japoneses ou Descendentes e Leucodermas): Contribuição ao Estudo. Tese de Doutorado, Londrina: Centro de Ciências da Saúde, Universidade Estadual de Londrina.

TEMPLETON, A. R., 1998. Human races: A genetic and evolutionary perspective. American Anthropologist, 100:632.

TOMITA, N., 1996. Relação entre Determinantes Sócio-Econômicos e Hábitos Bucais: Influência na Oclusão de Pré-Escolares de Bauru-SP, Brasil. Tese de Doutorado, Bauru: Faculdade de Odontologia de Bauru, Universidade de São Paulo.

TORRES, C. F. \& CORRALES, M. C., 1985. Prevalencia de maloclusiones en niños de 6 a 11 años de edad. Revista Cubana de Estomatología, 22:223-228.

TROMBELLI, L.; SALETTI, C.; VERNA, C. \& CALURA, G., 1991. Prevalenza de carie e malocclusioni in bambini in eta scolare della provincia di Ferrara. Mondo Ortodontico, 16:399-405.

TROTTMAN, A. \& ELSBACH, H. G., 1996. Comparison of malocclusion in preschool black and white children. American Journal of Orthodontics and Dentofacial Orthopedics, 110:69-72.

VAN DER LINDEN, F. P. G. M., 1966. Genetic and environmental factors in dentofacial morphology. American Journal of Orthodontics, 52:576-583.

VARRELA, J., 1990. Occurrence of malocclusion in attritive environment: A study of a skull sample from southwest Finland. Scandinavian Journal of Dental Research, 98:242-247.

VILLAVICENCIO, J. A. L.; FERNANDEZ, M. A. V. \& AHEDO, L. M., 1996. Ortopedia Dentofacial - Una Visión Multidisciplinaria. Caracas: Editorial A.M.D.

VISKOVIC, R.; VUJANOVIC, M. \& BRCIC, V., 1990. Prevalence of orthodontic anomalies, analysis and evaluation of dental health in three groups of pre-school children in Zadar. Acta Stomatologica Croatica, 24:271-280.

WEILAND, F. J.; JONKE, E. \& BANTLEON, H. P., 1997. Secular trends in malocclusion in Austrian men European Journal of Orthodontics, 19:355-359.

WHEELER, T. T.; McGORRAY, S. P.; YURKIEWICZ, L.; KEELING, S. D. \& KING, G. J., 1994. Orthodontic treatment demand and need in third and fourth grade schoolchildren. American Journal of Orthodontics and Dentofacial Orthopedics, 106:22-33.

Recebido em 30 de dezembro de 2000 Versão final reapresentada em 30 de julho de 2001 Aprovado em 15 de dezembro de 2001 